\title{
Improving glycemic control in critically ill patients: personalized care to mimic the endocrine pancreas
}

\author{
J. Geoffrey Chase ${ }^{1}$, Thomas Desaive ${ }^{2}$, Julien Bohe ${ }^{3}$, Miriam Cnop ${ }^{4}$, Christophe De Block ${ }^{5}$, Jan Gunst ${ }^{6}$, \\ Roman Hovorka ${ }^{7}$, Pierre Kalfon ${ }^{8}$, James Krinsley ${ }^{9}$, Eric Renard ${ }^{10}$ and Jean-Charles Preiser ${ }^{11^{*}}$
}

\begin{abstract}
There is considerable physiological and clinical evidence of harm and increased risk of death associated with dysglycemia in critical care. However, glycemic control (GC) currently leads to increased hypoglycemia, independently associated with a greater risk of death. Indeed, recent evidence suggests GC is difficult to safely and effectively achieve for all patients. In this review, leading experts in the field discuss this evidence and relevant data in diabetology, including the artificial pancreas, and suggest how safe, effective GC can be achieved in critically ill patients in ways seeking to mimic normal islet cell function. The review is structured around the specific clinical hurdles of: understanding the patient's metabolic state; designing GC to fit clinical practice, safety, efficacy, and workload; and the need for standardized metrics. These aspects are addressed by reviewing relevant recent advances in science and technology. Finally, we provide a set of concise recommendations to advance the safety, quality, consistency, and clinical uptake of GC in critical care. This review thus presents a roadmap toward better, more personalized metabolic care and improved patient outcomes.
\end{abstract}

Keywords: Glycemic control, Endocrine function, Artificial pancreas, Modeling, Model based, Validation, Virtual patient, In silico, Review

\section{Background}

Hyperglycemia is prevalent in critical care, caused by a complex interaction of multiple feedback loops associated with inflammation as a result of immune responses, counter-regulatory responses, and high blood glucose itself $[1,2]$. Hyperglycemia is exacerbated by unsuppressed endogenous glucose production [1], some medications (steroids/catecholamines), and high exogenously administered nutrition [3]. There is also suppression/loss of pancreatic insulin secretion, and loss of sensitivity to insulin, resulting in reduced insulin-mediated glucose uptake. Thus, the question arises of whether there is a need for an "artificial pancreas" or another form of closed-loop, highly personalized glycemic control (GC) in critical care, similar to those emerging in type 1 diabetes [4].

To date, blood glucose control to obtain metabolic homeostasis has given mixed results in clinical trials in

\footnotetext{
* Correspondence: Jean-Charles.Preiser@erasme.ulb.ac.be

${ }^{11}$ Department of Intensive Care, Erasme Hospital, Université Libre de Bruxelles, route de Lennik 808, 1070 Brussels, Belgium

Full list of author information is available at the end of the article
}

critically ill patients. Initial results of reduced morbidity and mortality with tight GC [5-9] could not be reproduced in large prospective trials [10-12]. More recent randomized trials using more advanced protocols have not altered the general direction [13]. However, recent observational analysis [14] indirectly supports the concept that altered glycemia, and not the underlying patient or metabolic condition, causes the increase in mortality, and thus GC is important and needs to be performed well. Thus, using higher blood glucose targets to ensure that hypoglycemia is avoided may not be good enough, reopening some of the debate on GC in terms of how to provide consistent, safe, effective management. However, significant issues prevent safe, effective GC, clinically, scientifically, and technologically-this short state-of-the-art review addresses these issues, resulting in a set of recommendations.

An effective GC protocol or artificial pancreas should provide insulin similar to a normal subject. In normoglycemic individuals, a hyperbolic relationship exists between insulin sensitivity and insulin secretion, leading to 
the disposition index concept, a measure of pancreatic beta cell function adjusted for insulin sensitivity [15]. Studies show that pancreatic function is deranged in critically ill patients [1] displaying similarities to type 2 diabetes [16, 17], namely insufficient insulin secretion in a context of decreased insulin sensitivity. The disposition index is therefore reduced, as in patients with diabetes, as a result of inflammatory and stress hormones. In both critical illness and type 2 diabetes, hyperglycemia results from reduction in the first-phase insulin response $[18,19]$. However, the associations between gene markers and outcome identified in type 2 diabetes have not been found in critical illness [20], and pancreatic function changes in critical illness are not associated with obesity or diet [21]. Thus, type 2 diabetes and critical care hyperglycemia can both feature reduced insulin sensitivity, reduced insulin clearance, and insufficiently increased insulin secretion, with resulting hyperglycemia. These effects can appear more severe over a very short term in critically ill patients and have different causes. In both conditions, the pancreas cannot provide the necessary insulin nor fully suppress hepatic glucose production.

The pancreas is linked to a continuous, accurate glucose "sensor" to guide insulin secretion and control. However, an equivalent "exogenous" sensor is lacking, despite emerging continuous glucose monitors [22]. Essential requirements for an artificial pancreas in the ICU include: accurate real-time or high-frequency continuous glucose monitoring [23]; continuous intravenous insulin infusion; and an adequate algorithm that automatically drives the intravenous insulin pump. A closed-loop system with accurate continuous glucose monitoring and computer-assisted titration of insulin based on glucose measurements could permit tight GC without increasing hypoglycemia and nursing staff workload.

However, there are significant hurdles to creating an effective artificial pancreas, both scientifically and technologically. A 2017 ISICEM Working Group addressing the artificial pancreas in critical care delineated and reviewed the recent research addressing these hurdles. The subsequent sections define the clinical hurdles in this field, which we use to define key scientific and technical needs. We then provide a short state-of-the-art overview for each need, leading to a set of recommendations for improving $\mathrm{GC}$ in the intensive care unit (ICU).

\section{Clinical hurdles}

Mixed results from GC clinical studies left the field questioning the weight of data correlating high glucose, high glycemic variability, and increased hypoglycemia from GC with increased morbidity and mortality. The result has led to a recommendation for a range of "soft" targets, essentially permitting hyperglycemia of 8.0-
$10.0 \mathrm{mmol} / \mathrm{l}$ to avoid hypoglycemia [24], despite evidence associating increased time in tight/intermediate glycemic bands with improved outcomes [25-27]. Hence, the field is at a crossroads between permissive hyperglycemia and the inability to safely, effectively control glycemia [14].

Indeed, safe strict GC has proved elusive $[5,7,9]$. Diverging results of randomized controlled trials on GC may be explained by important methodological differences between trials, compliance, experience, motivation, and/or protocol. Studies showing a positive impact of tight GC used accurate glucose monitors and were in general more successful in achieving the blood glucose target (e.g., [28]). In subsequent nonconfirmatory studies, the time in the target range was smaller. Achieving $>50 \%$ time in blood glucose bands of 4.0-7.0 and/or $5.0-8.0 \mathrm{mmol} / \mathrm{L}$ for $90 \%$ or more of patients is considered an index of good performance [25-27]. Although only some patients may benefit from good control [6], an inability to identify them directly requires safe, tight control to be delivered to all patients.

Recent work suggests that glycemic outcomes and their association with morbidity and mortality outcomes are a function of the quality of control applied [14]. While this analysis needs repeating over larger cohorts for certainty, it indicates that current acceptance of permissive hyperglycemia may carry risk and that GC has a role to play if it can be delivered with greater safety and performance. In particular, without better safety from hypoglycemia it is difficult to assess whether the effects of strict GC are beneficial. Personalized, patient-specific $\mathrm{GC}$, potentially including recognition of diabetic status or other factors, thus offers a route to GC that is safe and effective for all patients [29], which in turn would enable new trials to better assess benefit.

Personalized or patient-specific GC transforms bedside $\mathrm{GC}$ data into accurate representations of the patient-specific metabolic state. Patient-specific models can be used to safely design GC algorithms in silico, to minimize risk and avoid the mixed results arising from trial-and-error clinical protocol design.

Further clinical hurdles include ergonomics and workload [30, 31]. Poor ergonomics lead to noncompliance, and thus poor, inconsistent control and outcomes [30]. Workload, and thus the potential need for automation, further hinders clinical uptake. Emerging technologies with full automation of sensors and/or pumps offer first opportunities to examine the potential of automation on workload and GC quality [32].

A final hurdle is the inability to fully learn from prior efforts. Many studies do not report results in the same way, with numerous different metrics [33]. Those metrics reported often do not allow 
reconstruction of the full results distribution, limiting complete understanding. Hence, it is difficult to extract significant, generalizable lessons from many studies.

\section{Needs statement and goals}

These clinical hurdles yield four main needs:

A. To accurately understand patient-specific, realtime metabolic status.

B. To develop a validated means to design GC methods to fit clinical practice.

C. To create the right type of safe, effective GC, providing good control for all patients with an acceptable workload.

D. To determine the correct set of metrics to evaluate GC safety and performance, which is generalizable across studies.

These needs lead to three main goals to create a step-change in GC capability. Specifically, these are as follows:

1. Model and (virtual patient) simulator: addressing needs A and B using in-silico methods to safely design protocols and, in use, to personalize care to patient-specific metabolic status.
2. Control (approach): addressing need $\mathrm{C}$, where many approaches exist, both model-based and clinically derived, but few have provided consistent safety and performance.

3. Metrics: directly addressing need D and the difficulty in comparing the safety and performance across studies and protocols to derive the lessons learned and advance understanding.

Figure 1 outlines the current nurse in the loop approach to model-based GC. Replacing a model-based decision support method with a standard clinical protocol or nurse-driven care shows the loop used in today's standard care. Figure 2 shows how the three identified areas of need fit into this feedback loop in Fig. 1, and thus outline the overall review, as well as showing how these elements fit into clinical care.

\section{Models and virtual patient simulators \\ Metabolic system models}

There are several models of human metabolism, predominantly deterministic compartment models with specific terms representing the relevant physiological behaviors [34], as well as black-box or data-driven models with no direct physiological analogy or relevance. Given the need to identify the patient-specific metabolic status with limited data for personalized

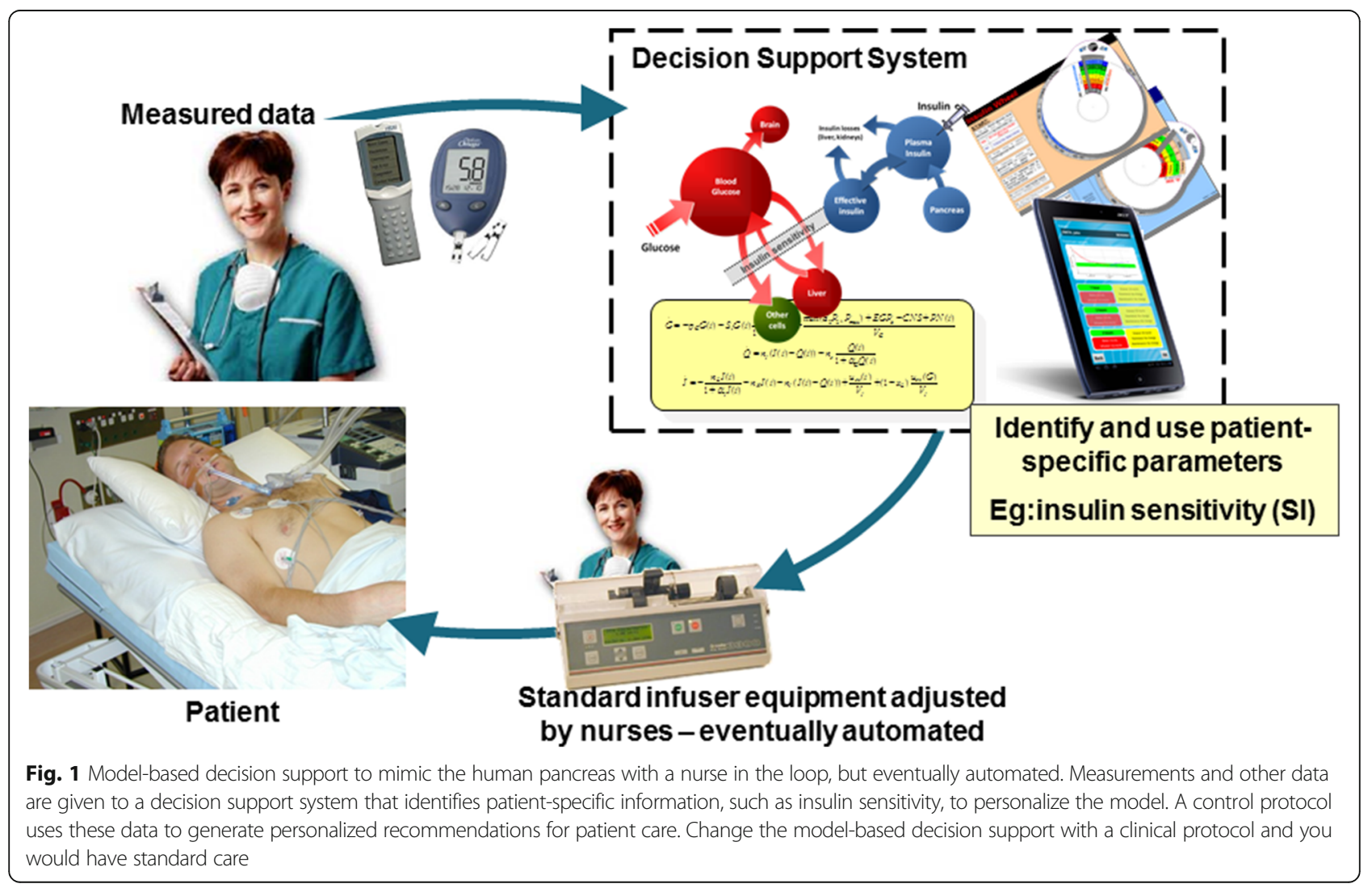




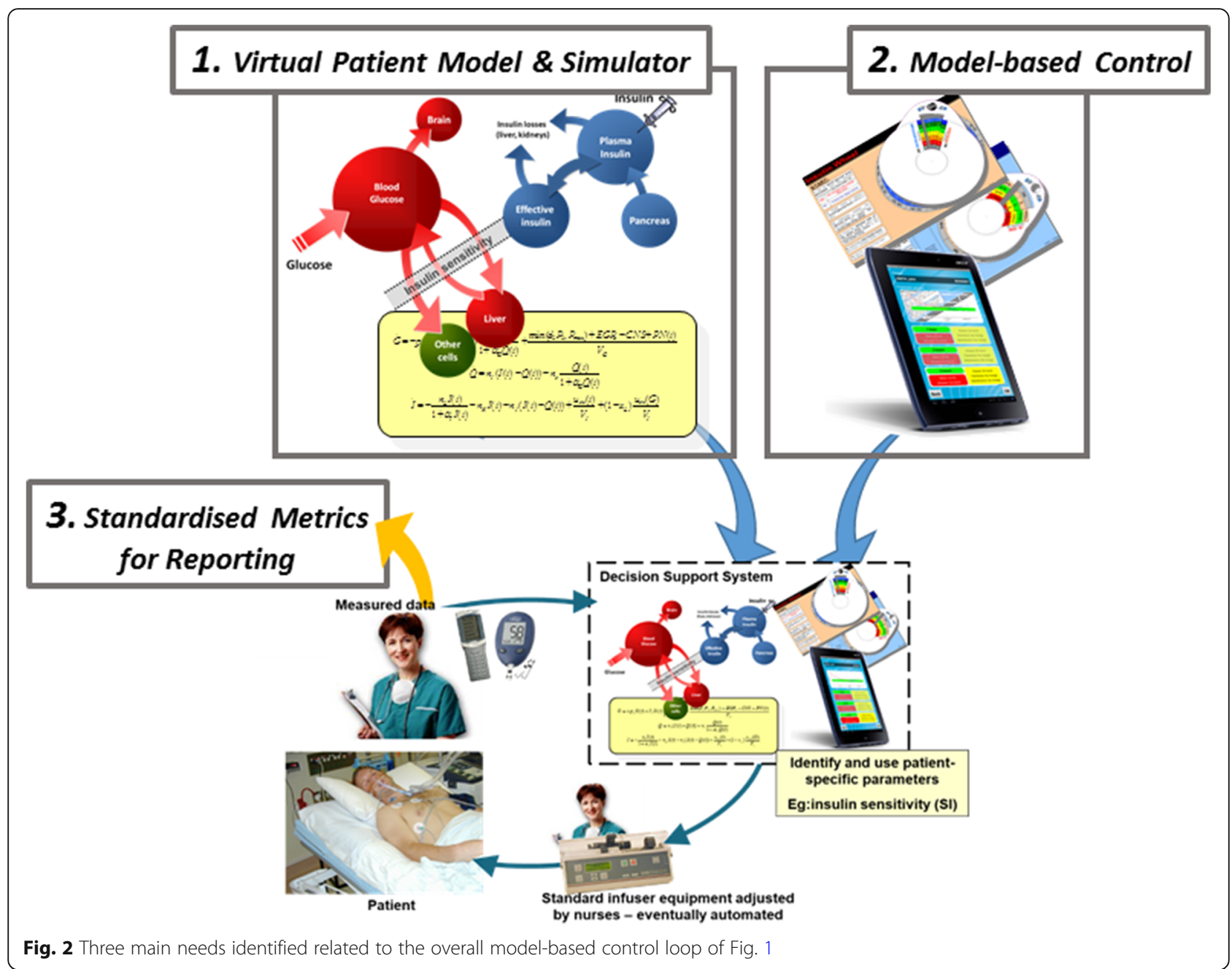

GC, this section focuses on less complex models, where the minimum fundamental model inputs to simulate and/or personalize these models are the insulin and nutrition given, and blood glucose measurements, but can be more extensive. However, no discussion of possible models is complete due to the large number of models that have been studied, so this section focuses on those used in critical care and for creating virtual patients, specifically:

- ICU Minimal Model [35].

- Glucosafe model [36].

- Cambridge model [37].

- ICING model [38, 39].

- UVA/Padova model [40].

Each model has been used to create in-silico virtual patients, and has thus moved beyond limited clinical experiments toward engineering design tools to optimize care.
The ICU Minimal Model is based on the Bergman Minimal Model [41], and was used to design the LOGIC controller [42]. It is the least physiologically relevant, with a minimal number of terms aggregating all glucose appearance and disappearance routes, and thus the least specific in terms of how the model terms directly relate to specific physiological phenomena. Identifying an insulin sensitivity parameter derived from two model parameters makes it patient specific. It has been used clinically and has shown improved control over nurse-managed $\mathrm{GC}$, a final form of validation [43].

Glucosafe is derived from an earlier form of the ICING model and a diabetes model [44]. Insulin sensitivity is identified from data and used to create virtual patients [36]. Glucosafe is more physiologically relevant than the ICU Minimal Model. However, it has demonstrated limited performance in pilot GC trials [45].

The Cambridge model is highly physiologically relevant [37], was developed from frequently sampled bedside data collected in the ICU, and has been used to 
simulate and design GC in critical care. It uses identified time-variant insulin sensitivity to guide care and mitigate errors [46].

The ICING model also has high physiological relevance, has an insulin sensitivity parameter identifiable from bedside data, and has been used in virtual patients $[39,47], \mathrm{GC}$ design $[48,49]$, and real-time GC $[49,50]$.

The UVA/Padova simulator was developed for type 1 diabetes [40]. This is not a single model, but a collection of submodels with very high physiological relevance and detail. However, a given insulin sensitivity or other key parameters cannot necessarily be identified from bedside data, limiting it to simulations of generic patients, and it is thus not validated for clinical use.

While this brief overview is not exhaustive, it clearly highlights the need for "valid models" meeting three main criteria. First, an identifiable insulin sensitivity parameter capturing patient-specific glucose responses to insulin/nutrition inputs to enable personalization. Second, a large enough degree of physiological relevance to ensure that the identified parameter(s) accurately capture patient-specific behavior so they can be used to design and/or guide care. Third, rigorous validation via use in the design and/or implementation of safe, effective GC. These issues are inter-related, and thus reviewed in subsequent sections in terms of the use of these models as virtual patients to design and guide care, and their subsequent use in guiding GC.

\section{Virtual patients and simulators}

Given a metabolic model and the required insulin, nutrition, and blood glucose data to identify key parameters to personalize the model, a virtual patient might be created. A virtual patient is built from the combination of a metabolic model and clinical patient data, creating an in-silico representation of that patient on which new treatment approaches might be tested, either to create a new protocol and/or in real time at the bedside to guide care safely and effectively. Thus, a metabolic model captures behavior, and a virtual patient simulator uses that model with clinical data to mimic patient behavior to design new therapeutic approaches.

Virtual patients come in two types:

- Generic/cohort specific: created from clinical data or model parameter choices, they capture overall patient types or behaviors.

- Clones/patient specific: created directly from clinical data, they capture the actual patient-specific response of a specific patient, and have thus created an in-silico model "clone" of the patient from their clinical data, which responds and acts in the same way as the actual patient.
Generic virtual patients use a selection of clinical data to fit general, cohort-wide model parameters, which are perturbed to create realistic cohorts. Simulations are only valid for predicting cohort-level responses, such as average glycemia and variability, safety from hypoglycemia, and required insulin/nutrition inputs. However, specific virtual patient results may not be realistic. Examples include the Cambridge simulator [37, 51], the Zealand simulator [52], the UVA/Padova simulator [53], the Medtronic simulator [54], and the ICU Minimal Model simulator [35] in diabetes. The Cambridge model has captured the central tendency of an ICU cohort [55].

Cloning specific patients from their data to create an in-silico, computer model mimic is rarer. The UVA model was used for single-day clones [56] and the Cambridge model in multiday clones $[37,51,55]$ in critical care. Glucosafe [36] was used to create multiday ICU patient clones for clinical GC [45]; however, the clinical outcomes did not match reality as well as desired. Finally, the ICING model and predecessors were used to create multiday virtual patient clones to design and implement multiple GC protocols. Predicted cohort-level glycemic results were matched by subsequent clinical results (e.g., [49]) with good correlation of median per-patient glucose levels, indicating that the cloned virtual patients were a good representation [39, 47].

The primary concern in creating virtual patients to design GC protocols is whether results of the virtual patient simulations accurately predict clinical safety, performance, and workload when a given protocol is implemented clinically. Accurate prediction of what happens when a model is implemented clinically provides assurance that the model and virtual patient simulator have the fidelity to design a protocol with confidence in the resulting safety, performance, and workload, as well as the fidelity to be used in real-time GC in decision support. Only the Cambridge and ICING simulators have delivered good results in this regard. To date, only the ICING and Cambridge models have been validated using independent cohorts and protocols [39, 47, 55].

A GC protocol design with virtual patients can be highly effective at predicting GC safety and performance in clinical use. It can thus limit poor results, particularly from easily avoidable protocol design errors. The main hurdle is the low number of validated models available.

\section{Control}

\section{Glycemic control and decision support}

GC using semi-automated (human-in-the-loop) or fully automated insulin/nutrition dosing is a long-term research area. Increasing automation brings technological risk, but significantly reduces workload, human error, and compliance risks. However, both approaches use the same GC protocol, so this section focuses on the two 
main means of applying control. Specifically, looking backward using a feedback approach, and looking forward using a predictive approach. Both approaches can be personalized using models, although personalization is typically only used in predictive methods.

\section{Feedback control}

Feedback control takes blood glucose measurements and other inputs, and suggests interventions in response to the current state and/or changes since the last measurement/intervention.

The most well-known feedback control systems are paper-based or computerized clinical protocols, typically ranging from simple static sliding scales to more complex dynamic scales. Static scales adjust insulin based on the blood glucose level alone. They are the simplest systems and offer no adaptability, with a "one size fits all" approach, thus essentially assuming a linear effect of increasing insulin with all patients assumed to have similar insulin sensitivity [57]. Dynamic scales change the insulin dose, based on the blood glucose range the patient is in and prior responses. The Leuven [7], Krinsley [9], and NICE-SUGAR [11] protocols are dynamic scales. However, many do not directly consider or control for nutrition, creating significant variation in responses [58].

Proportional-derivative (PD) and/or proportional-integral-derivative (PID) controllers operate like complex dynamic scales. Each insulin intervention is based on the error in blood glucose from the target $(\mathrm{P})$, the rate of change of that error (D), and the area under the error curve (I). The P, I, and D gains multiply these values using the most recent blood glucose measurements to calculate the insulin dose. PID control and similar dynamic scales in critical care include the Endotool (Monarch Medical, USA) [59], GRIP [60], Glucommander (Glytec Systems, USA) [61], GlucoCare (Pronia Medical, USA) [62], and GlucoStabiliser (Medical Decision Network, USA) [63] systems commercially and several research tools [13, 14, 64-66]. Advantages include simplicity and easy automation. Disadvantages include lack of significant patient specificity, which is only seen indirectly via patient-specific glucose response to insulin, and lack of input about nutrition or (often) prior insulin doses. These disadvantages can lead to greater variability across patients in larger, heterogeneous cohorts.

Overall, the performance of clinical protocols or dynamic scales is better than that of static sliding scales, but is exceeded by PID control. More successful protocols include modifications around insulin on board, patient-specific response, and/or physiological inputs. While these models improve safety and performance, they have similar increased clinical workload to systems without automation.
Unlike all other feedback controllers, SPRINT [5] controls nutrition. Developed using virtual trials [47, 48], this protocol modulates insulin and nutrition using implicitly calculated patient-specific insulin sensitivity. SPRINT is thus more patient specific and was the only protocol associated with reduced glycemia, mortality, and hypoglycemia. The main message from this study [5] was the need to consider nutrition in safe, effective GC, which model-based, predictive controllers can do.

\section{Predictive control}

Predictive control requires a model that takes current measurements and other inputs, identifies patient-specific model parameters to personalize the control, and uses the patient-specific model to predict the outcome of insulin/nutrition interventions to optimize glycemic performance. The model is used directly in the control loop.

Although several model-based and predictive controllers have been tested in short or limited trials, only a few have been used regularly in major trials [42] or as a standard of care $[50,67]$. There are two essential approaches: target to value (TTV), GC to a specified glycemic target value; and target to range or risk (TTR), GC to a specified risk of hypoglycemia or hyperglycemia.

The LOGIC-Insulin system is a TTV approach and nutrition is clinically set and not controlled. The randomized, single-center LOGIC-I trial [42] compared standard care at a unit with a good reputation for nursing-led GC to LOGIC-Insulin model-based care with very good results. This performance was confirmed in a multicenter trial [43], but it is not yet a standard of care in the original study unit.

The TTV eMPC (B. Braun, Germany) [46] has been used in several trials [66-69], controlling insulin infusions and leaving nutrition clinically set. Compared to standard care, eMPC does well [66, 69]. Comparisons across centers and cohorts show similar, but not identical, performance [67, 68]. Workload is $14-18$ measurements/day, and thus higher than standard care. Thus, eMPC provides improved care and safety, but increases potential workload. It is used regularly in some ICUs.

STAR is the only TTR system and controls both insulin and nutrition input $[49,70]$, using risk-based stochastic forecasting [71]. It is the only GC system to directly account for future variability. STAR has good performance and safety, including high times in intermediate glycemic bands, approaching $80 \%$ with $10-13$ measurements/day [50]. It generalized well, with almost identical glycemic outcomes across very different cohorts and ICUs [50]. Notably, only eMPC and LOGIC-Insulin have shown similar generalizability.

All three model-based systems noted have very low rates of hypoglycemia ( $<5 \%$ by patient) and are very 
generalizable. These results indicate that predictive, model-based methods can overcome many of the hurdles that have hindered several other trials.

In summary, at this time, in addition to PID feedback systems used in some hospitals in the USA [61-63], three model-based predictive GC methods have proved reliable over multiple patient types and centers. Two methods are used as standards of care in multiple hospitals, and one of these has a neonatal ICU version [72] used as standard care and in a randomized trial [73]. Both methods consider nutrition, although only one controls it, which may account for some differences in performance. Overall, successful model-based GC takes into account patient-specific factors. The patient's metabolic state evolves over the course of their illness, and thus this approach provides adjustments as needed within a given patient, as much as across patients.

\section{Metrics and a recommended minimum standard}

Glycemic reporting metrics are diverse [33]. Given significant analyses linking organ failure and mortality to time in target bands [6, 25, 27], time-in-band percentages should be standard cohort and patient-level metrics. Unlike the median (IQR) or mean (standard deviation), time in range to a given threshold (e.g., patients with $>50 \%$ blood glucose within $4.0-7.0 \mathrm{mmol} / \mathrm{L}$ ) captures the central tendency (mean or median) and variability (IQR, standard deviation). Higher thresholds indicate tighter control for a given cohort or patient in a given blood glucose range.

To assess any range of the several possible, the cohort cumulative distribution function (CDF) or s-curve should be reported. This approach enables any time in range to be assessed. These CDFs can be aggregated for each patient, and provide a data set with every possible time-in-range outcome shown, from which comparisons can be readily made.

For safety, the percentage blood glucose $<3.9 \mathrm{mmol} / \mathrm{L}$ and the percentage of patients who have one or more episodes of blood glucose $<2.2 \mathrm{mmol} / \mathrm{L}$ should be reported.

Workload can be minimally reported as measurements/day, because measurements are a primary source of workload [31], but could add other GC-related effort [30].

All of these metrics would include all patients for all days of stay, from the start of GC. These recommendations provide a minimal data set for regular and standardized comparison across cohorts, trials, and publications. Any other analyses or data reporting would be additional to this minimal set.

\section{Summary recommendations}

Based on this overview and analysis of the current state-of-the-art for GC in critically ill patients, the following recommendations are made to advance the safety, quality, consistency, and clinical uptake:

1. Patient-specific model-based GC including closedloop systems, increasingly enabled by the penetration of computational technology into the ICU, can improve the quality of GC:

a. models should be self-validated and crossvalidated;

b. initial assessment and optimization in validated virtual trials should be considered for new GC methods;

c. final validation of safety and performance must come in clinical (pilot and/or randomized) trials.

2. GC could consider nutrition as an input certainly, and possibly as a controlled clinical input set against international guideline goal feeds. No GC care should be "carbohydrate blind", even though closed-loop systems may operate without nutritional input.

3. No GC trial should be performed without first conducting a pilot trial in each involved ICU, which demonstrates that safe, effective GC can be obtained to the desired level for $80 \%$ or more of patients, unless an algorithm is used that has already been validated in a multicenter context. Each potential trial center must prove it can safely and consistently achieve the desired level of GC stated in the trial plan.

4. To enable comparison and analysis, all GC reporting should have a minimum standardized set of data reporting performance, safety, and workload, including:

a. performance-time in desired target band;

b. safety-number of patients experiencing severe hypoglycemia (blood glucose $<2.2 \mathrm{mmol} / \mathrm{L}$ ) or percentage blood glucose $<3.9 \mathrm{mmol} / \mathrm{L}$;

c. workload-average number of staff-taken or automated (if applicable) glucose measurements per patient per day (standard deviation) or median (IQR);

d. performance (optional)-CDFs of cohort glycemia, which provide all possible time-intarget ranges, enabling far easier comparison;

e. performance (optional)-CDFs of per-patient glycemia.

5. ICU clinicians should press for increasing automation and access to sensor and infusion pump data for independent processing of GC methods to increase safety and reduce workload.

\section{Conclusions}

Glycemic control has proven difficult to safely and effectively achieve for all patients, where modeling and 
model-based methods have offered a potentially significant avenue to achieving safe, effective control. In this review, leading experts discuss this evidence, report relevant reports from medicine and engineering, and suggest how safe, effective GC can be achieved in critically ill patients, ultimately seeking to mimic pancreatic function. The review concludes with concise recommendations to advance the safety, quality, consistency, and clinical uptake of GC in critical care, providing a roadmap toward better, more personalized metabolic care and improved patient outcomes.

\section{Abbreviations}

CDF: Cumulative distribution function; GC: Glucose control; ICU: Intensive care unit; TTR: Target to range or risk; TTV: Target to value

\section{Funding}

This document was supported by unrestricted educational grants from BBraun, GlucoSet, Menarini Diagnostics, Nikkiso, Roche Pharma, Nova Biomedical, and Sphere. These companies had no influence on the content of the manuscript or on the decision to publish.

\section{Authors' contributions}

JGC, TD, and J-CP developed the first draft of the manuscript based on presentations by all of the authors at a meeting on glucose control endorsed by the Diabetes Technology Society and the European Society of Intensive Care Medicine. JB, MC, CDB, JG, RH, PK, JK, and ER critically reviewed and revised the article for important intellectual content. All authors read and approved the final manuscript.

\section{Ethics approval and consent to participate}

Not applicable.

\section{Consent for publication}

Not applicable.

\section{Competing interests}

JGC has consulted for Medtronic and Monarch Medical. CDB is a consultant for Abbott, A. Menarini Diagnostics, Medtronic, and Roche Diagnostics. RH reports having received speaker honoraria from Eli Lilly, Novo Nordisk, and Astra Zeneca, serving on advisory panels for Eli Lilly and Novo Nordisk, receiving license fees from BBraun and Medtronic, and having served as a consultant to BBraun. JK is a consultant for Edwards, Medtronic, Roche Diagnostics, and Optiscan. J-CP is a consultant for Edwards, Medtronic, and Optiscan, and is an Associate Editor for Critical Care. The remaining authors declare that they have no conflicts of interest relevant to this article.

\section{Publisher's Note}

Springer Nature remains neutral with regard to jurisdictional claims in published maps and institutional affiliations.

\section{Author details}

'Department of Mechanical Engineering, Centre for Bio-Engineering, University of Canterbury, Christchurch, New Zealand. ${ }^{2}$ GIGA In-Silico Medicine, University of Liège, Liège, Belgium. ${ }^{3}$ Medical Intensive Care Unit, Lyon-Sud University Hospital, Pierre-Bénite, France. ${ }^{4}$ ULB Center for Diabetes Research, and Division of Endocrinology, Erasme Hospital, Université Libre de Bruxelles, Brussels, Belgium. ${ }^{5}$ Department of Endocrinology, Diabetology and Metabolism, Antwerp University Hospital, Edegem, Belgium. ${ }^{6}$ Clinical Division and Laboratory of Intensive Care Medicine, Department of Cellular and Molecular Medicine, KU Leuven, Leuven, Belgium. 'University of Cambridge Metabolic Research Laboratories, Level 4, Wellcome Trust-MRC Institute of Metabolic Science, Addenbrooke's Hospital, Cambridge, UK. ${ }^{8}$ Service de Réanimation polyvalente, Hôpital Louis Pasteur, CH de Chartres, Chartres, France. ${ }^{9}$ Division of Critical Care, Department of Medicine, Stamford Hospital, Columbia University College of Physicians and Surgeons, Stamford, CT, USA

${ }^{10}$ Department of Endocrinology, Diabetes, Nutrition, and Institute of
Functional Genomics, CNRS, INSERM, Montpellier University Hospital, University of Montpellier, Montpellier, France. ${ }^{11}$ Department of Intensive Care, Erasme Hospital, Université Libre de Bruxelles, route de Lennik 808, 1070 Brussels, Belgium.

Received: 8 March 2018 Accepted: 29 June 2018

Published online: 02 August 2018

\section{References}

1. Dungan KM, Braithwaite SS, Preiser JC. Stress hyperglycaemia. Lancet. 2009; 373:1798-807.

2. Marik PE, Raghavan M. Stress-hyperglycemia, insulin and immunomodulation in sepsis. Intensive Care Med. 2004;30:748-56.

3. Rice TW. Gluttony in the intensive care unit: time to push back from the consensus table. Am J Respir Crit Care Med. 2013;187:223-4.

4. Renard E, Farret A, Kropff J, Bruttomesso D, Messori M, Place J, et al. Dayand-night closed-loop glucose control in patients with type 1 diabetes under free-living conditions: results of a single-arm 1-month experience compared with a previously reported feasibility study of evening and night at home. Diabetes Care. 2016:39:1151-60.

5. Chase JG, Shaw G, Le Compte A, Lonergan T, Willacy M, Wong XW, et al. Implementation and evaluation of the SPRINT protocol for tight glycaemic control in critically ill patients: a clinical practice change. Crit Care. 2008;12:R49.

6. Chase JG, Pretty CG, Pfeifer L, Shaw GM, Preiser JC, Le Compte AJ, et al. Organ failure and tight glycemic control in the SPRINT study. Crit Care. 2010;14:R154.

7. van den Berghe $G$, Wouters P, Weekers F, Verwaest C, Bruyninckx F, Schetz M, et al. Intensive insulin therapy in critically ill patients. N Engl J Med. 2001; 345:1359-67.

8. van den Berghe G, Wilmer A, Hermans G, Meersseman W, Wouters PJ, Milants I, et al. Intensive insulin therapy in the medical ICU. N Engl J Med. 2006;354:449-61.

9. Krinsley JS. Effect of an intensive glucose management protocol on the mortality of critically ill adult patients. Mayo Clin Proc. 2004;79:992-1000.

10. Brunkhorst FM, Engel C, Bloos F, Meier-Hellmann A, Ragaller M, Weiler N, et al. Intensive insulin therapy and pentastarch resuscitation in severe sepsis. N Engl J Med. 2008;358:125-39.

11. Finfer S, Chittock DR, Su SY, Blair D, Foster D, Dhingra V, et al. Intensive versus conventional glucose control in critically ill patients. N Engl J Med. 2009;360:1283-97.

12. Preiser JC, Devos P, Ruiz-Santana S, Melot C, Annane D, Groeneveld J, et al. A prospective randomised multi-centre controlled trial on tight glucose control by intensive insulin therapy in adult intensive care units: the Glucontrol study. Intensive Care Med. 2009;35:1738-48.

13. Kalfon P, Giraudeau B, Ichai C, Guerrini A, Brechot N, Cinotti R, et al. Tight computerized versus conventional glucose control in the ICU: a randomized controlled trial. Intensive Care Med. 2014:40:171-81.

14. Uyttendaele V, Dickson JL, Shaw GM, Desaive T, Chase JG. Untangling glycaemia and mortality in critical care. Crit Care. 2017;21:152.

15. Kahn SE, Prigeon RL, McCulloch DK, Boyko EJ, Bergman RN, Schwartz MW, et al. Quantification of the relationship between insulin sensitivity and betacell function in human subjects. Evidence for a hyperbolic function. Diabetes. 1993;42:1663-72.

16. Cnop M, Igoillo-Esteve M, Rai M, Begu A, Serroukh Y, Depondt C, et al. Central role and mechanisms of beta-cell dysfunction and death in friedreich ataxia-associated diabetes. Ann Neurol. 2012;72:971-82.

17. Ferrannini E, Gastaldelli A, Miyazaki Y, Matsuda M, Mari A, DeFronzo RA. Beta-cell function in subjects spanning the range from normal glucose tolerance to overt diabetes: a new analysis. J Clin Endocrinol Metab. 2005; 90:493-500.

18. Luzi $L$, DeFronzo RA. Effect of loss of first-phase insulin secretion on hepatic glucose production and tissue glucose disposal in humans. Am J Phys. 1989;257:E241-6.

19. Basu A, Dalla MC, Basu R, Toffolo G, Cobelli C, Rizza RA. Effects of type 2 diabetes on insulin secretion, insulin action, glucose effectiveness, and postprandial glucose metabolism. Diabetes Care. 2009;32:866-72.

20. Morris AP, Voight BF, Teslovich TM, Ferreira T, Segre AV, Steinthorsdottir V, et al. Large-scale association analysis provides insights into the genetic architecture and pathophysiology of type 2 diabetes. Nat Genet. 2012;44: 981-90. 
21. Ahren B. Type 2 diabetes, insulin secretion and beta-cell mass. Curr Mol Med. 2005;5:275-86.

22. Preiser JC, Chase JG, Hovorka R, Joseph JI, Krinsley JS, De Block C, et al. Glucose control in the ICU: a continuing story. J Diabetes Sci Technol. 2016;10:1372-81.

23. Krinsley JS, Chase JG, Gunst J, Martensson J, Schultz MJ, Taccone FS, et al. Continuous glucose monitoring in the ICU: clinical considerations and consensus. Crit Care. 2017:21:197.

24. Moghissi ES, Korytkowski MT, DiNardo M, Einhorn D, Hellman R, Hirsch $\mathrm{IB}$, et al. American Association of Clinical Endocrinologists and American Diabetes Association consensus statement on inpatient glycemic control. Diabetes Care. 2009;32:1119-31.

25. Krinsley JS, Preiser JC. Time in blood glucose range 70 to $140 \mathrm{mg} / \mathrm{dl}$ $>80 \%$ is strongly associated with increased survival in non-diabetic critically ill adults. Crit Care. 2015;19:179.

26. Penning S, Pretty C, Preiser JC, Shaw GM, Desaive T, Chase JG. Glucose control positively influences patient outcome: a retrospective study. J Crit Care. 2015;30:455-9.

27. Signal M, Le Compte A, Shaw GM, Chase JG. Glycemic levels in critically ill patients: are normoglycemia and low variability associated with improved outcomes? J Diabetes Sci Technol. 2012;6:1030-7.

28. Okabayashi T, Shima Y, Sumiyoshi T, Kozuki A, Tokumaru T, liyama T, et al. Intensive versus intermediate glucose control in surgical intensive care unit patients. Diabetes Care. 2014;37:1516-24.

29. Chase JG, Le Compte AJ, Preiser JC, Shaw GM, Penning S, Desaive T. Physiological modeling, tight glycemic control, and the ICU clinician: what are models and how can they affect practice? Ann Intensive Care. 2011;1:11.

30. Chase JG, Andreassen S, Jensen K, Shaw GM. Impact of human factors on clinical protocol performance: a proposed assessment framework and case examples. J Diabetes Sci Technol. 2008;2:409-16.

31. Aragon D. Evaluation of nursing work effort and perceptions about blood glucose testing in tight glycemic control. Am J Crit Care. 2006; 15:370-7.

32. Okabayashi T, Kozuki A, Sumiyoshi T, Shima Y. Technical challenges and clinical outcomes of using a closed-loop glycemic control system in the hospital. J Diabetes Sci Technol. 2013;7:238-46.

33. Eslami S, de Keizer NF, de Jonge E, Schultz MJ, Abu-Hanna A. A systematic review on quality indicators for tight glycaemic control in critically ill patients: need for an unambiguous indicator reference subset. Crit Care. 2008;12:R139.

34. Carson ER, Cobelli C. Modelling methodology for physiology and medicine. Amsterdam: Elsevier; 2001.

35. Van Herpe T, Espinoza M, Haverbeke N, Moor BD, van den Berghe G. Glycemia prediction in critically ill patients using an adaptive modeling approach. J Diabetes Sci Technol. 2007;1:348-56.

36. Pielmeier U, Andreassen S, Nielsen BS, Chase JG, Haure P. A simulation model of insulin saturation and glucose balance for glycemic control in ICU patients. Comput Methods Prog Biomed. 2010;97:211-22

37. Hovorka R, Chassin LJ, Ellmerer M, Plank J, Wilinska ME. A simulation model of glucose regulation in the critically ill. Physiol Meas. 2008;29:959-78.

38. Lin J, Razak NN, Pretty CG, Le Compte A, Docherty P, Parente JD, et al. A physiological Intensive Control Insulin-Nutrition-Glucose (ICING) model validated in critically ill patients. Comput Methods Prog Biomed. 2011;102: 192-205.

39. Dickson JL, Stewart KW, Pretty CG, Flechet M, Desaive T, Penning S, et al. Generalisability of a virtual trials method for glycaemic control in intensive care. IEEE Trans Biomed Eng. 2018;65:1543-1553.

40. Kovatchev BP, Breton M, Man CD, Cobelli C. In silico preclinical trials: a proof of concept in closed-loop control of type 1 diabetes. J Diabetes Sci Technol. 2009:3:44-55.

41. Bergman RN, Finegood DT, Ader M. Assessment of insulin sensitivity in vivo. Endocr Rev. 1985;6:45-86.

42. Van Herpe T, Mesotten D, Wouters PJ, Herbots J, Voets E, Buyens J, et al. LOGIC-insulin algorithm-guided versus nurse-directed blood glucose control during critical illness: the LOGIC-1 single-center, randomized, controlled clinical trial. Diabetes Care. 2013;36:188-94.

43. Dubois J, Van Herpe T, van Hooijdonk RT, Wouters R, Coart D, Wouters P, et al. Software-guided versus nurse-directed blood glucose control in critically ill patients: the LOGIC-2 multicenter randomized controlled clinical trial. Crit Care. 2017;21:212
44. Arleth $\mathrm{T}$, Andreassen S, Federici MO, Benedetti MM. A model of the endogenous glucose balance incorporating the characteristics of glucose transporters. Comput Methods Prog Biomed. 2000;62:219-34.

45. Pielmeier U, Andreassen S, Juliussen B, Chase JG, Nielsen BS, Haure P. The Glucosafe system for tight glycemic control in critical care: a pilot evaluation study. J Crit Care. 2010;25:97-104.

46. Hovorka R, Kremen J, Blaha J, Matias M, Anderlova K, Bosanska L, et al. Blood glucose control by a model predictive control algorithm with variable sampling rate versus a routine glucose management protocol in cardiac surgery patients: a randomized controlled trial. J Clin Endocrinol Metab. 2007;92:2960-4.

47. Chase JG, Suhaimi F, Penning S, Preiser JC, Le Compte AJ, Lin J, et al. Validation of a model-based virtual trials method for tight glycemic control in intensive care. Biomed Eng Online. 2010;9:84.

48. Lonergan $\mathrm{T}$, Le Compte A, Willacy M, Chase JG, Shaw GM, Wong XW et al. A simple insulin-nutrition protocol for tight glycemic control in critical illness: development and protocol comparison. Diabetes Technol Ther. 2006:8:191-206

49. Fisk LM, Le Compte AJ, Shaw GM, Penning S, Desaive T, Chase JG. STAR development and protocol comparison. IEEE Trans Biomed Eng. 2012:59:3357-64.

50. Stewart KW, Pretty CG, Tomlinson H, Thomas FL, Homlok J, Noemi SN, et al. Safety, efficacy and clinical generalization of the STAR protocol: a retrospective analysis. Ann Intensive Care. 2016;6:24.

51. Wilinska ME, Chassin LJ, Hovorka R. In silico testing-impact on the progress of the closed loop insulin infusion for critically ill patients project. J Diabetes Sci Technol. 2008;2:417-23.

52. Wendt SL, Ranjan A, Moller JK, Schmidt S, Knudsen CB, Holst JJ, et al. Cross-validation of a glucose-insulin-glucagon pharmacodynamics model for simulation using data from patients with type 1 diabetes. J Diabetes Sci Technol. 2017;11:1101-11

53. Visentin R, Giegerich $C$, Jager R, Dahmen R, Boss A, Grant M, et al. Improving efficacy of inhaled technosphere insulin (Afrezza) by postmeal dosing: in-silico clinical trial with the University of Virginia/ Padova type 1 diabetes simulator. Diabetes Technol Ther. 2016;18:5 74-85.

54. Kanderian SS, Weinzimer SA, Steil GM. The identifiable virtual patient model: comparison of simulation and clinical closed-loop study results. J Diabetes Sci Technol. 2012;6:371-9.

55. Wilinska ME, Blaha J, Chassin LJ, Cordingley JJ, Dormand NC, Ellmerer M et al. Evaluating glycemic control algorithms by computer simulations. Diabetes Technol Ther. 2011;13:713-22.

56. Visentin R, Man CD, Cobelli C. One-day Bayesian cloning of type 1 diabetes subjects: toward a single-day UVA/Padova type 1 diabetes simulator. IEEE Trans Biomed Eng. 2016;63:2416-24.

57. Le Compte AJ, Pretty CG, Lin J, Shaw GM, Lynn A, Chase JG. Impact of variation in patient response on model-based control of glycaemia in critically ill patients. Comput Methods Prog Biomed. 2013;109:211-9.

58. Chase JG, Le Compte AJ, Suhaimi F, Shaw GM, Lynn A, Lin J, et al. Tight glycemic control in critical care-the leading role of insulin sensitivity and patient variability: a review and model-based analysis. Comput Methods Prog Biomed. 2011;102:156-71.

59. Tanenberg RJ, Hardee S, Rothermel C, Drake AJ III. Use of a computerguided glucose management system to improve glycemic control and address national quality measures: a 7-year, retrospective observational study at a tertiary care teaching hospital. Endocr Pract. 2017;23:331-41.

60. Vogelzang M, Zijlstra F, Nijsten MW. Design and implementation of GRIP: a computerized glucose control system at a surgical intensive care unit. BMC Med Inform Decis Mak. 2005;5:38.

61. Davidson PC, Steed RD, Bode BW. Glucommander: a computer-directed intravenous insulin system shown to be safe, simple, and effective in 120,618 h of operation. Diabetes Care. 2005;28:2418-23.

62. Marvin MR, Inzucchi SE, Besterman BJ. Computerization of the Yale insulin infusion protocol and potential insights into causes of hypoglycemia with intravenous insulin. Diabetes Technol Ther. 2013;15:246-52.

63. Juneja R, Golas AA, Carroll J, Nelson D, Abad VJ, Roudebush CP, et al. Safety and effectiveness of a computerized subcutaneous insulin program to treat inpatient hyperglycemia. J Diabetes Sci Technol. 2008;2:384-91.

64. Meijering S, Corstjens AM, Tulleken JE, Meertens JH, Zijlstra JG, Ligtenberg JJ. Towards a feasible algorithm for tight glycaemic control in critically ill patients: a systematic review of the literature. Crit Care. 2006;10:R19. 
65. Shetty S, Inzucchi SE, Goldberg PA, Cooper D, Siegel MD, Honiden S. Adapting to the new consensus guidelines for managing hyperglycemia during critical illness: the updated Yale insulin infusion protocol. Endocr Pract. 2012:18:363-70.

66. Amrein K, Ellmerer M, Hovorka R, Kachel N, Parcz D, Korsatko S, et al. Hospital glucose control: safe and reliable glycemic control using enhanced model predictive control algorithm in medical intensive care unit patients. Diabetes Technol Ther. 2010;12:405-12.

67. Blaha J, Barteczko-Grajek B, Berezowicz P, Charvat J, Chvojka J, Grau T, et al. Space GlucoseControl system for blood glucose control in intensive care patients - a European multicentre observational study. BMC Anesthesiol. 2016;16:8

68. Blaha J, Kopecky P, Matias M, Hovorka R, Kunstyr J, Kotulak T, et al. Comparison of three protocols for tight glycemic control in cardiac surgery patients. Diabetes Care. 2009:32:757-61.

69. Pachler C, Plank J, Weinhandl H, Chassin LJ, Wilinska ME, Kulnik R, et al. Tight glycaemic control by an automated algorithm with time-variant sampling in medical ICU patients. Intensive Care Med. 2008;34:1224-30.

70. Evans A, Shaw GM, Le Compte A, Tan CS, Ward L, Steel J, et al. Pilot proof of concept clinical trials of Stochastic Targeted (STAR) glycemic control. Ann Intensive Care. 2011;1:38.

71. Lin J, Lee D, Chase JG, Shaw GM, Le Compte A, Lotz T, et al. Stochastic modelling of insulin sensitivity and adaptive glycemic control for critical care. Comput Methods Prog Biomed. 2008:89:141-52.

72. Dickson JL, Chase JG, Lynn A, Shaw GM. Model-based glycaemic control: methodology and initial results from neonatal intensive care. Biomed Tech (Berl). 2017;62:225-33.

73. Alsweiler J, Williamson K, Bloomfield F, Chase G, Harding J. Computer-determined dosage of insulin in the management of neonatal hyperglycaemia (HINT2): protocol of a randomised controlled trial. BMJ Open. 2017;7:e012982.

Ready to submit your research? Choose BMC and benefit from:

- fast, convenient online submission

- thorough peer review by experienced researchers in your field

- rapid publication on acceptance

- support for research data, including large and complex data types

- gold Open Access which fosters wider collaboration and increased citations

- maximum visibility for your research: over $100 \mathrm{M}$ website views per year

At BMC, research is always in progress.

Learn more biomedcentral.com/submissions 